\title{
Galectin-1 has potential prognostic significance and is implicated in clear cell renal cell carcinoma progression through the HIF/mTOR signaling axis
}

\author{
N M A White ${ }^{1,2}$, O Masui ${ }^{3}$, D Newsted ${ }^{1}$, A Scorilas ${ }^{4}$, A D Romaschin ${ }^{1,2}$, G A Bjarnason ${ }^{5}, K$ W M Siu ${ }^{3}$ \\ and G M Yousef ${ }^{\star}, 2,3$
}

${ }^{1}$ The Keenan Research Center in the Li Ka Shing Knowledge Institute and the Department of Laboratory Medicine, St Michael's Hospital, Toronto, Ontario M5B 1W8, Canada; ${ }^{2}$ Department of Laboratory Medicine and Pathobiology, University of Toronto, Toronto, Ontario M5S 1A8, Canada; ${ }^{3}$ Department of Chemistry and Centre for Research in Mass Spectrometry, 4700 Keele Street York University, Toronto, Ontario M3J 1P3, Canada; ${ }^{4}$ Department of Biochemistry and Molecular Biology, University of Athens, Panepistimiopolis, Athens 157 01, Greece and ${ }^{5}$ Medical Oncology, Sunnybrook Odette Cancer Centre, Toronto, Ontario M4N 3M5, Canada

Background: Metastatic clear cell renal cell carcinoma (ccRCC) patients have $<9 \% 5$-year survival rate, do not respond well to targeted therapy and eventually develop resistance. A better understanding of molecular pathways of RCC metastasis is the basis for the discovery of novel prognostic markers and targeted therapies.

Methods: We investigated the biological impact of galectin-1 (Gal-1) in RCC cell lines by migration and invasion assays. Effect of Gal-1 expression on the mitogen-activated protein kinase pathway was assessed by proteome array.

Results: Increased expression of Gal-1 increased cell migration while knocking down Gal-1 expression by siRNA resulted in reduced cellular migration $(P<0.001)$ and invasion $(P<0.05)$. Gal-1 overexpression increased phosphorylation of Akt, $m T O R$ and p70 kinase. Upon hypoxia and increased HIF-1 $\alpha$, Gal-1 increased in a dose-dependent manner. We also found miR-22 overexpression resulted in decreased Gal-1 and HIF-1 $\alpha$. Immunohistochemistry analysis showed that high Gal-1 protein expression was associated with larger size tumor $(P=0.034)$, grades III/IV tumors $(P<0.001)$ and shorter disease-free survival $(P=0.0013)$. Using the Cancer Genome Atlas data set, we found that high Gal-1 mRNA expression was associated with shorter overall survival (41 vs 78 months; $P<0.01$ ).

Conclusions: Our data suggest Gal-1 mediates migration and invasion through the HIF-1 $\alpha$-mTOR signaling axis and is a potential prognostic marker and therapeutic target.

Galectins are a family of 15 carbohydrate-binding proteins that have affinity for $\beta$-galactoiside (Barondes et al, 1994). Galectin-1 (Gal-1), encoded by the LGALS1 gene, is widely expressed in tissues and exerts pleiotropic biological functions. It can exist both extra- and intracellularly and has different functions depending on its cellular location. When found extracellularly, Gal-1 can bind to cell-surface receptors including integrins (Romaniuk et al, 2012) as well as members of the extracellular matrix (van den Brule et al, 2003). Intracellularly, Gal-1 can interact with different proteins and has been shown to contribute to cancer pathogenesis through 
pathways involved in immune suppression (Brandt et al, 2010), angiogenesis (Croci et al, 2012) and metastasis (Jung et al, 2008). Gal-1 expression was shown to be associated with tumor progression in oral squamous cell carcinoma (Chiang et al, 2008), cervical (Kim et al, 2012a), ovarian (Kim et al, 2012b) and breast (Jung et al, 2007) cancers, among others.

There has been little reported about the involvement of Gal-1 in clear cell renal cell carcinoma (ccRCC). Clear cell renal cell carcinoma is the most common type of kidney cancer in adults and is among the top 10 most prevalent malignancies in North America. Due to the asymptomatic nature of early RCC, a proportion of patients present with metastatic disease. Metastatic RCC is difficult to treat and patients have a dismal prognosis with $<9 \% 5$-year survival rate. Understanding the mechanisms of RCC metastasis will help identify the molecules that contribute to disease aggressiveness and will also help identify new potential candidates/pathways for targeted therapy. Molecular profiling holds much promise in this regard (Arsanious et al, 2009; White and Yousef, 2011) and could pave the way to a new era of personalised medicine in kidney cancer (Diamandis et al, 2010).

Only one group other than ours have reported the dysregulation of Gal-1 in RCC. Dihazi et al (2007) reported Gal-1 differential expression in RCC cells compared with normal kidney cell lines. More recently, we showed, using quantitative proteomics analysis, that Gal-1 had significant increased expression in metastatic and primary RCC tumors when compared with normal kidney tissues and represents a potential prognostic marker for RCC patients (Masui et al, 2013). In this study, we explore the involvement of Gal-1 in RCC tumor progression and metastasis. Using RCC cell line models, we show that Gal-1 can affect RCC cellular migration and invasion and is involved in the HIF/mTOR signaling axis. We also show that Gal-1 expression can be induced by HIF- $1 \alpha$ and that miR-22 can target Gal-1 both directly and indirectly through HIF1- $\alpha$. Moreover, we show that Gal-1 is a potential marker of poor prognosis in ccRCC.

\section{MATERIALS AND METHODS}

Patient specimens and protein extraction. We analyzed 102 ccRCC patients in this study. Primary ccRCC tissues and matched normal kidney tissue from the same patient, as well as metastatic RCC tissues were obtained from St Michael's Hospital and the Ontario Tumor Bank, Toronto, Canada. All specimens were histologically confirmed by a pathologist. The study was approved by the Research Ethics Boards of St Michael's Hospital. In addition, we analyzed gene expression data from 404 ccRCC patients using the publically available database, the Cancer Genome Atlas (http://cancergenome.nih.gov/).

Tissues were prepared as described previously (Ralhan et al, 2008). Briefly, tissues were washed three times in ice-cold phosphate-buffered saline (PBS) and homogenised using a handheld homogeniser in a protease inhibitor cocktail (Roche, Laval, Canada). Cell debris was then removed by centrifugation at $4{ }^{\circ} \mathrm{C}$ for $30 \mathrm{~min}$ at 14000 r.p.m. The clarified supernatant was transferred to a fresh tube and protein concentration was determined using the BCA method (Pierce Biotechnology, Rockford, IL, USA).

Western blot analysis. Dysregulated protein expression in metastatic RCC samples was verified by western blot (WB) analysis. Briefly, $30 \mu \mathrm{g}$ of total protein were electrophoretically separated on a SDS-PAGE gel. Proteins were transferred to a nitrocellulose membrane and probed with primary antibodies overnight at $4{ }^{\circ} \mathrm{C}$. Primary antibodies were used for Gal-1 (Abcam, Cambridge, MA, USA), HIF- $1 \alpha$ (Novus Biologicals, Littleton, CO, USA), phospho-mTOR S2448 (serine 2448), total mTOR,
phospho-Akt S473 (serine 473), total Akt and $\alpha$-tubulin (Cell Signaling Technology, Danvers, MA, USA) was used as a loading control. The following day, membranes were washed with trisbuffered saline and Tween-20 and incubated with a secondary antirabbit antibody conjugated with horseradish peroxidase. Finally, membranes were incubated with enhanced chemoluminescence reagent (Amersham Pharmacia Biotech, Piscataway, NJ, USA) and protein expression was visualised after exposure to X-ray film. Each experiment was performed in triplicate and representative blots are shown. Densitometry analysis was performed using Image J software from the National Institutes of Health (http://rsbweb.nih. gov/ij/) and differences were evaluated using the Student's $t$-test.

RNA extraction and real-time PCR. Total RNA was extracted from cell lysate using RNeasy (Qiagen, Mississauga, ON, Canada) according to the manufacturer's protocol. Total RNA concentrations were determined spectrophotometrically and the quality of extracted RNA was assessed using the Agilent 2100 Bioanalyzer (Agilent, Santa Clara, CA, USA). Samples were stored at $-80^{\circ} \mathrm{C}$ and used for further analysis only if they had a RNA integrity number $\geqslant 8.0$.

One microgram total RNA was reversed transcribed using the High Capacity cDNA Reverse Transcription Kit (Life Technologies, Grand Island, NY, USA). Gene expression was measured for LGALS1 and peptidylprolyl isomerase A (PPIA) was used as an endogenous control on the Step One Plus Real-Time PCR System (Life Technologies). Primers sequences were: LGALS1, F - 5'-CAAA CCTGGAGAGTGCCTTC-3', R - 5'-GATGCACACCTCTGCA ACAC-3'; and PPIA, F - 5'-ATGCTGGCCCCAACACAA- $3^{\prime}$, $\mathrm{R}$ - 5'-CCCTCTTTCACCTTGCCACC-3'.

Cell culture and plasmid transfection. 786-O, ACHN and CAKI-1 RCC cell lines were obtained from American Type Culture Collection (ATCC; Manassas, VA, USA). 786-O and ACHN cell lines were maintained in Dulbecco's modified Eagle's medium (DMEM; Wisent Inc., St Bruno, Quebec, Canada) supplemented with $10 \%$ foetal calf serum (FCS). CAKI-1 cells were maintained in McCoy's 5A medium (Wisent Inc.) supplemented with $10 \%$ FCS. All cells were maintained in $5 \% \mathrm{CO}_{2}$ at $37^{\circ} \mathrm{C}$.

The LGALS1 sequence (NM_002305.3) was inserted into the multiple cloning site of the pcDNA3.1 plasmid between the BamHI and EcoRV restriction sites by Gene Art Gene Synthesis (Life Technologies). Proper insertion was confirmed with sequencing. Plasmids were transfected using Lipofectamine 2000 (Life Technologies). One microgram plasmid DNA was mixed with $100 \mu \mathrm{l}$ Opti-MEM I Reduced Serum Medium (Life Technologies). In a separate tube, $3 \mu \mathrm{l}$ Lipofectamine 2000 was added to $100 \mu \mathrm{l}$ Opti-MEM I Reduced Serum Medium. After 5-min incubation, the two tubes were mixed and incubated at room temperature for $20 \mathrm{~min}$. Complexes were then added to cell medium and incubated at $37^{\circ} \mathrm{C}$ with $5 \% \mathrm{CO}_{2}$. Akt phosphorylation was inhibited using LY294002 (Cell Signaling Technology).

Loss-of-function experiments. Three separate ON-TARGET plus (Dharmacon RNAi Technologies, Lafayette, CO, USA) siRNAs directed against LGALS1 and a control, non-targeting siRNA, were resuspended as suggested by the manufacturer. For siRNA transfection, cells were plated in antibiotic-free medium and allowed to adhere overnight. The following day, $5 \mu \mathrm{m}$ siRNA was diluted in Opti-MEM I Reduced Serum Medium (Life Technologies). In a separate tube, DharmaFECT transfection reagent (Dharmacon RNAi Technologies) was diluted in Opti-MEM I Reduced Serum Medium (Life Technologies) and allowed to incubate at room temperature for $5 \mathrm{~min}$. Diluted siRNAs and transfection reagents were then mixed and complexes were allowed to form for $20 \mathrm{~min}$. Complexes were then added to the cell medium and incubated at $37^{\circ} \mathrm{C}$ and $5 \% \mathrm{CO}_{2}$. 
Migration assay. $786-\mathrm{O}$ cells were plated at $8.0 \times 10^{4}$ cells per well in a 12-well plate and transfected with siRNA directed against LGALS1 or a non-targeting/control siRNA as described above. Twenty-four hours after transfection, the cell monolayer was wounded with a $200 \mu \mathrm{l}$ pipette tip. Photomicrographs were taken at the time of wounding $(0 \mathrm{~h})$ and $11 \mathrm{~h}$ later. The rate of migration is displayed as the percentage of cell covered area (100-percent cellfree area) where percent cell-free area is defined as ((cell-free area $_{11 \mathrm{~h}} /$ cell-free $\left.\left.\operatorname{area}_{0 \mathrm{~h}}\right) \times 100\right)$. Area was measured using Image Software (http://rsbweb.nih.gov/ij/). Cells were transfected in three separate experiments and each was analyzed in triplicate. There were three images taken per 'wound'.

CAKI-1 cell migration was measured by comparing the number of cells that migrated through the BD BioCoat $8.0 \mu \mathrm{m}$ polyethylene terephthalate membrane (BD BioSciences, Mississauga, ON, Canada) before and after transfection. Cells were transfected in three separate experiments.

Invasion assay. The effect of Gal-1 knockdown on cellular invasion was examined using BD BioCoat Matrigel Invasion Chambers (BD Biosciences). 786-O cells were transfected with siRNA as described above and $24 \mathrm{~h}$ after transfection cells were trypsinised and resuspended in DMEM supplemented with $0.5 \%$ FCS. A total of $5.0 \times 10^{4}$ cells were plated on the upper chamber. Normal growth medium, DMEM supplemented with 10\% FCS, was added to the bottom chamber as a chemoattractant. Cells were allowed to invade for $22 \mathrm{~h}$. After incubation, non-invading cells were removed from the upper surface and cells on the lower surface were stained with Diff-Quick (Siemens, Burlington, ON, Canada). Membranes were photographed and invading cells were assayed by taking the average of the number of cells at $\times 40$ magnification in three fields. The experiment was performed in triplicate. Cell invasion is displayed as \% invasion (mean number of cells that invaded through Matrigel insert/mean number of cells that migrated through the control insert membrane).

Proteome profiler array. The Human Phospho-MAPK Array Kit (R\&D Systems, Minneapolis, MN, USA) was used to assay for relative levels of phosphorylation (and not total protein) of mitogen-activated protein kinases (MAPKs) and other serine/ threonine kinases before and after treatment. Briefly, cells were rinsed with PBS three times and solubilised with Lysis Buffer 6 as supplied by the manufacturer. Supernatants were cleared by centrifugation at $14000 \mathrm{~g}$ for $10 \mathrm{~min}$ at $4{ }^{\circ} \mathrm{C}$. Protein concentrations were determined with the BCA Protein Assay Reagent (Pierce Biotechnology) using bovine serum albumin as a standard. Capture and control antibodies were spotted in duplicate on nitrocellulose membranes. After blocking for $1 \mathrm{~h}, 200 \mu \mathrm{g}$ total protein from untreated and treated cells was added to each of the membranes and incubated overnight at $4{ }^{\circ} \mathrm{C}$. The following day, both membranes were washed three times for $10 \mathrm{~min}$ each. Membranes were then incubated in streptavidin-HRP for $30 \mathrm{~min}$ followed by three washes of $10 \mathrm{~min}$ each. Chemi Reagent Mix, as supplied by the manufacturer, was added to the membranes for $1 \mathrm{~min}$. Membranes were then exposed to X-ray film and densitometry, as described above, was performed to determine intensity. Images were adjusted for differences in background between membranes. Changes in phosphorylation levels were calculated as fold change $=$ (average intensity $_{(\text {transfected cells) }} /$ average intensity $\left.{ }_{(\text {control cells) }}\right)$.

Immunoflorescence confocal images. Cells were cultured in fourwell chamber slides and transfection was carried out as described above. Forty-eight hours after transfection, cells were washed with PBS (pH 7.4) three times and fixed in 4\% p-formaldehyde solution for $15 \mathrm{~min}$. Cells were then washed with PBS and incubated in $0.1 \%$ Triton X-100 for $10 \mathrm{~min}$. After washing with PBS, nonspecific binding was blocked with 1-h incubation in 1\% BSA in PBS. Cells were then incubated with anti-vimentin conjugated with Alexa-488
(Cell Signaling Technology) in a humid chamber for $1 \mathrm{~h}$ followed by washing with PBS. For actin staining, cells were incubated with rhodamine phalloidin conjugate (Molecular Probes, Grand Island, NY, USA) in a humid chamber for $45 \mathrm{~min}$. Finally, cells were washed in PBS and mounted. Confocal microscopy was performed with a Zeiss LSM 700 confocal microscope (Carl Zeiss Microimaging Inc., Oberkochen, Germany) equipped with a Plan-Apochromat $63 \times / 1.4$ oil objective lens. The confocal images were acquired using Zen 2010 software (Carl Zeiss Microimaging Inc.).

Prognostic significance and statistical analyses. In order to assess prognostic ability, we examined Gal-1 protein expression on a tissue microarray of 92 cases of primary ccRCC using immunohistochemistry and correlated expression with diseasefree and overall survival. Based on expression in the cytoplasm, patients were divided into two groups: low Gal-1 expression $(<50 \%$ tumor cells show Gal-1 positivity) and high Gal-1 expression ( $>50 \%$ tumor cells are positive for Gal-1; van den Brule et al, 2003). We correlated Gal-1 expression with clinic parameters including sex, tumor size, tumor grade and tumor stage, using the $\chi^{2}$ test and with survival using Cox hazard regression and Kaplan-Meier analyses.

Data of gene expression levels for LGALS1 were obtained from 404 patients of primary ccRCC using 'level 3' gene expression data (normalised gene expression data derived from the Cancer Genome Characterization Center (CGCC) at the University of North Carolina (unc.edu) using the Illumina HiSeq RNA Sequencing platform) for LGALS1 in ccRCC. Overall survival data were obtained from the Cancer Genome Atlas (TCGA), available through the cBio Cancer Genomics Portal (www.cbioportal.org/ public-portal/). The X-tile algorithm was used to generate a prognostic optimal cutoff point to dichotomise LGALS1 mRNA expression as 'LGALS1 - low expression' and 'LGALS1 - high expression' using Monte Carlo $P$-value $<0.05$.

Significant differences in migration and invasion assays were analyzed using one-way ANOVA. Differences between each condition were assessed using Tukey's multiple comparisons test.

\section{RESULTS}

Validation of Gal-1 overexpression in metastatic RCC. We recently identified proteins significantly dysregulated in metastatic RCC (Masui et al, 2013). Our results showed that Gal-1 had increased expression in metastatic when compared with primary ccRCC. To validate these results, we compared Gal-1 protein expression in an independent set of 20 primary and metastatic ccRCC patients. We found Gal-1 was significantly upregulated in metastatic compared with primary tumors at both the mRNA (Figure 1A), as measured by real time PCR, and protein (Figure 1B) levels, shown by WB. We also examined Gal-1 expression in RCC cell lines. The 786-O and ACHN cell lines had high protein expression levels of Gal-1 while the CAKI-1 cell line had no detectable expression when measured by WB analysis (Figure 1C).

Gal-1 promotes migratory ability of RCC cells. In order to see if Gal-1 has a direct effect on cellular migration, we transfected CAKI-1 cells with pLGALS1, the gene coding for Gal-1. After transfection with pLGALS1, there was significant increased Gal-1 protein expression as expected (Figure 1D). We then measured the rate of cellular migration before and after transfection. We found CAKI-1 cells transfected with pLGALS1 showed an increased rate of cellular migration when compared with control cells or cells transfected with the empty plasmid $(P<0.01$; Figure $1 E)$. We also examined whether Gal-1 had any effect on the distribution of F-actin and vimentin after transfection using immunofluorescence. We found that untransfected RCC cells showed diffuse expression of actin and vimentin (Figures $1 \mathrm{~F}-\mathrm{H}$ ). On the other hand, cells transfected with the pLGALS1 plasmid showed that expression of 


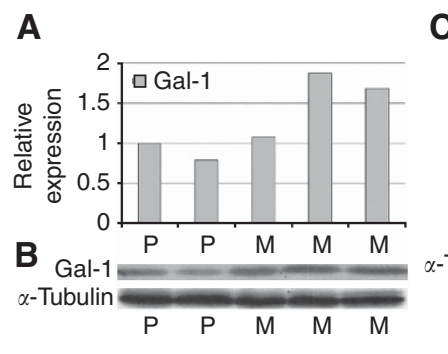

C
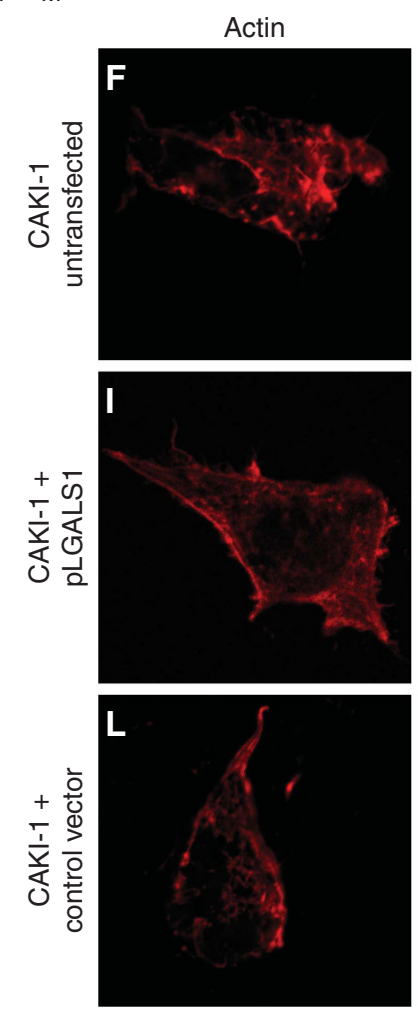

D

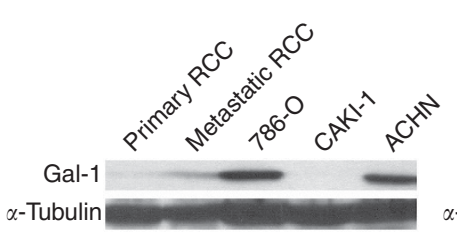

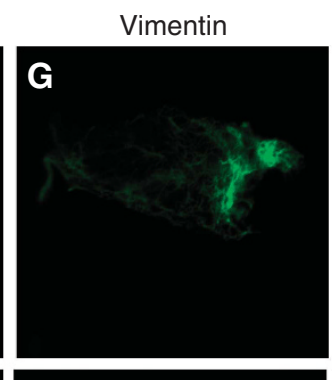
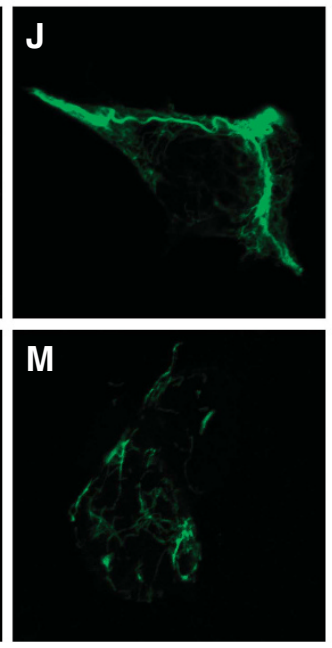
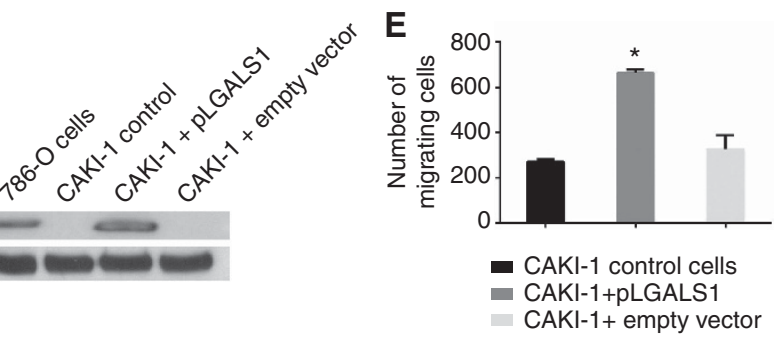
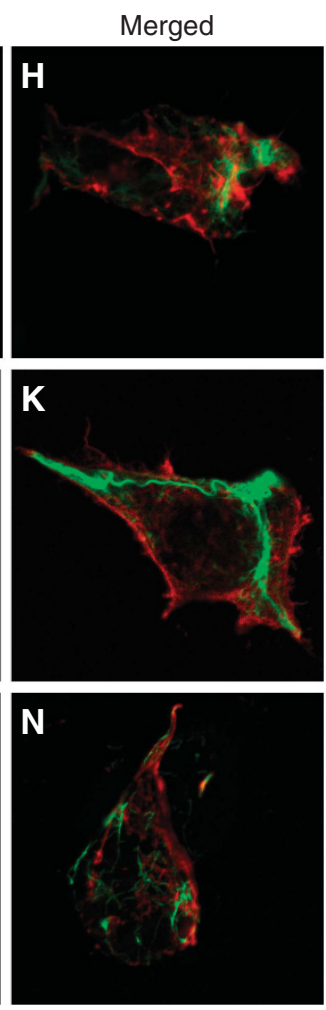

Figure 1. (A) Real time PCR showing increased Gal-1 mRNA expression in metastatic RCC when compared to primary RCC tissues. (B) A representative WB analysis showing increased expression of Gal-1 protein in metastatic RCC (M), compared with primary tumors (P). (C) WB analysis showing the expression of Gal-1 in RCC cell lines. Both 786-O and ACHN cell lines had higher expression levels of Gal-1, whereas expression was undetectable in CAKI-1 cells. $\alpha$-Tubulin was used as a loading control. (D) Validation of successful Gal-1 transfection in CAKI-1 cells is shown by WB. High Gal-1 protein expression was observed in cells after transfection with the pLGALS1 plasmid compared with untransfected cells. Cells transfected with the empty vector had undetectable levels of Gal-1 protein. 786-O cells were used as a positive control and $\alpha$-tubulin was used as a loading control. (E) Transfection of Gal-1 resulted in increased cellular migration rate in CAKI-1 cells as measured using transwell inserts. A significantly higher number of CAKI-1 cells transfected with pLGALS1 migrated across the membrane when compared with untransfected cells or cells transfected with the empty vector. ${ }^{*} P<0.01$. (F-H) F-actin and vimentin showed diffuse expression in the CAKI-1 control cells. (I-K) CAKI-1 cells transfected with pLGALS1 showed expression of F-actin and vimentin concentrated along the leading edge of the cell. (L-N) CAKI-1 cells transfected with the control vector showed expression patterns comparable to the control cells. Images are representative of at least three independent experiments. Cells were visualized by laser scanning confocal microscope (Zeiss LSM 700, objective plan-apochromat $63 \times / 1.4$, oil immersion).

actin (Figure 1I) and vimentin (Figures 1J and K) were concentrated along the leading edge of the cell. Cells that were transfected with the control vector showed expression patterns comparable to the untransfected cells (Figures 1L-N).

The effect of Gal-1 loss-of-function on cancer cell behaviour. To further validate our findings, we examined if targeted knockdown of Gal-1 expression would have any potential anticancer effects. We transfected the 786-O cell line, which has high Gal-1 expression (Figure 1C), with three separate siRNAs directed against LGALS1. siRNA knockdown resulted in significant decreases of Gal-1 expression at both the mRNA (Figure 2A) and protein (Figure 2B) levels with all three siRNAs $(P<0.01)$. Cells transfected with a non-targeting siRNA or transfection reagent alone showed no significant difference in Gal-1 expression when compared with untransfected cells.
We assayed for cellular migration before and after transfection with siRNA using a wound-healing assay. Cells transfected with siRNA directed against LGALS1 migrated significantly slower than control cells $(P<0.01$; Figures $2 \mathrm{C}$ and $\mathrm{D})$ while there was no significant change in migration when cells were transfected with a non-targeting siRNA or Dharmafect. In addition, we performed invasion assays, using matrigel covered transwell inserts, to determine if Gal-1 expression had an effect on the invasive ability of cells. We found that cells transfected with any of the three LGALS1 siRNAs showed a significant reduction in cellular invasion compared with control cells (Figure 2E). This same effect was not seen when cells were transfected with transfection reagent-only control or a nontargeting siRNA. On the other hand, there was no significant effect of LGALS1 knockdown on cellular proliferation (data not shown). 
A

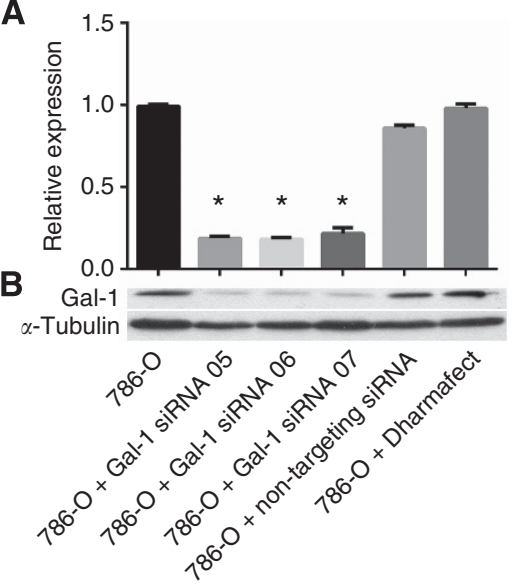

C

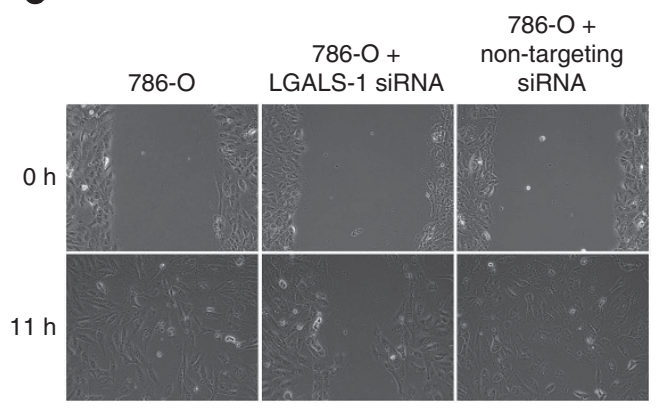

D

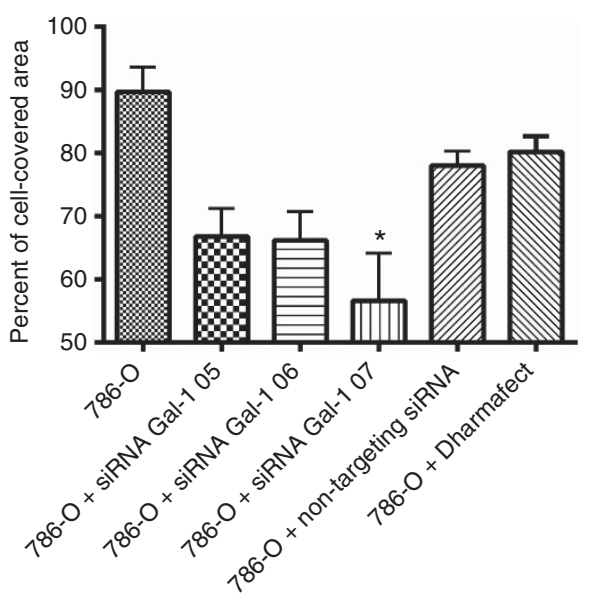

E

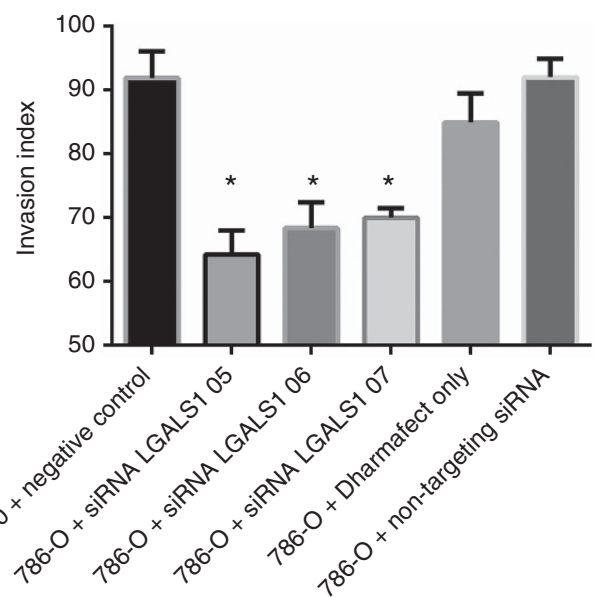

Figure 2. Transfection of 786-O cells with three separate siRNAs directed against LGALS1 resulted in significant decrease of Gal-1 expression at both the $(\mathbf{A})$ mRNA, and $(\mathbf{B})$ protein levels $\left({ }^{\star} P<0.01\right)$. (C) A representative photomicrograph showing cellular migration at the time of wounding $(0 \mathrm{~h})$ and $11 \mathrm{~h}$ later. 786-O cells transfected with LGALS1 siRNA had had a slower migration rate than untransfected cells or cells transfected with a non-targeting siRNA. (D) A bar graph summarizing the results of the wound-healing assay. Cells transfected with siRNA directed towards LGALS1 showed slower rate of migration compared with cells transfected with the non-targeting siRNA or untransfected cells (*P<0.01).

(E) Cellular invasion was measured using transwell chamber assays. Cells that were transfected with either of the three siRNAs directed against LGALS1 had a significantly lower invasion index when compared with that of untransfected cells and cells transfected with non-targeting siRNA or vehicle only. ${ }^{\star} P<0.01$.

Gal-1 is a downstream effector molecule of HIF-1 $\alpha$. Gal-1 was previously shown to be regulated by hypoxia, and it was suggested to be under transcriptional control of HIF- $1 \alpha$ in colon cancer (Zhao et al, 2010). To examine the hypothesis that Gal-1 is a downstream effector of HIF- $1 \alpha$ in RCC, we induced HIF- $1 \alpha$ expression by adding $\mathrm{CoCl}_{2}$ to cell medium. $\mathrm{CoCl}_{2}$ mimics hypoxia by inhibiting prolyl hydroxylase and subsequently, ubiquitination of HIF- $1 \alpha$, resulting in increased levels of HIF- $1 \alpha$ (Ho and Bunn, 1996). We found HIF- $1 \alpha$ expression was increased in a dosedependent manner by $4.5,7.7,8.7,14.9$ times $(P<0.001)$, in CAK-1 cells after the addition of 100, 200, 300 and $400 \mu \mathrm{M} \mathrm{CoCl}_{2}$, respectively (Figures $3 \mathrm{~A}$ and $\mathrm{B}$ ). A similar dose-dependent increase in Gal-1 protein expression by $6.1,11.3,11.1$ and 14.0 times, respectively, as shown by densitometry $(P<0.001$, Figure $3 \mathrm{~B})$ was also observed. These results suggest Gal-1 is a transcriptional target of HIF- $1 \alpha$ in RCC cells.

Exploring the mechanisms for Gal-1-mediated cellular migration and invasion in RCC. In order to determine the downstream molecular pathways affected by Gal-1 overexpression, we compared the expression of members of the MAPK pathway before and after Gal-1 transfection using a MAPK protein array. We observed an increase in the phosphorylation of a number of proteins in cells that were transfected with pLGALS1 when compared with untransfected cells (Figures 3C and D). Overall, $38 \%$ (10 out of 26 ) proteins showed increased phosphorylation after transfection with pLGALS1, whereas the remaining 62\% (16 out of 26) showed phosphorylation levels that were unchanged. There were no proteins that showed decreased phosphorylation after pLGALS1 transfection. Proteins that showed the most significant increases include Akt pan S473, S474, S472 (2.2 times increased expression), GSK-3 $\beta$ S9 (2.1 times increased expression), JNK3 T221/Y223 (1.8 times increased expression) and mTOR S2448 (1.4 times increased expression). Interestingly, many of these proteins are involved in cell adhesion and motility.

Of special interest is the activation of mTOR, as currently a number of targeted therapies for metastatic RCC are directed towards the mTOR signaling pathway. We further validated the involvement of Gal-1 in the PI3K/Akt and mTOR signaling pathways using WB analysis (Figure 3E). Cells were transfected with pLGALS1 and siRNA directed towards LGALS1 and immunoblotted for phosphorylated Akt and mTOR. We found that after transfection with pLGALS1, cells showed increased phosphorylation of both Akt (S473) and mTOR (S2448). This effect was not observed after transfection with the control vector and was abolished with the addition of siRNA directed towards 
A

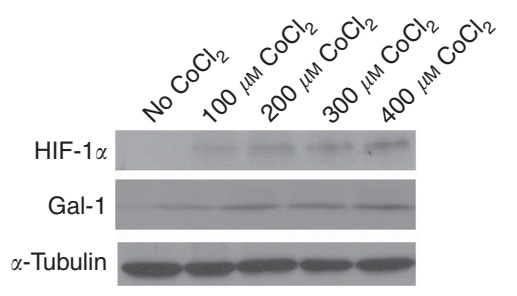

D

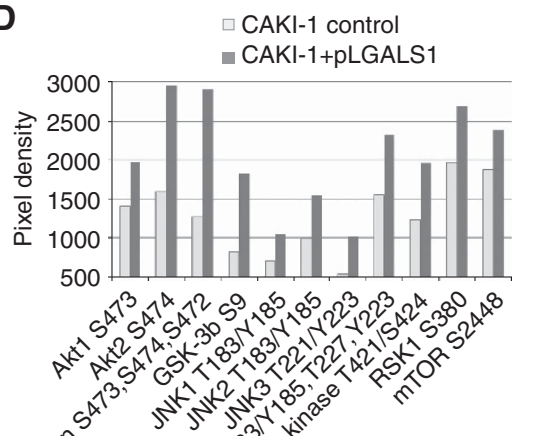

B

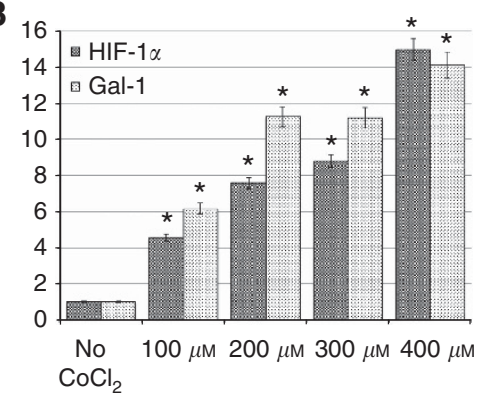

E

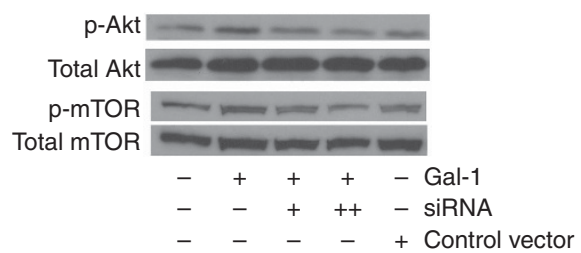

C

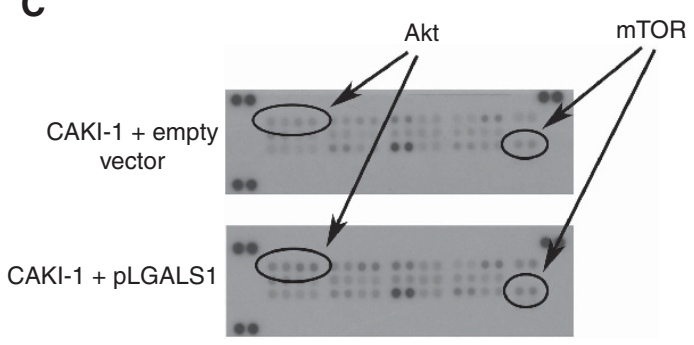

$\mathbf{F}$

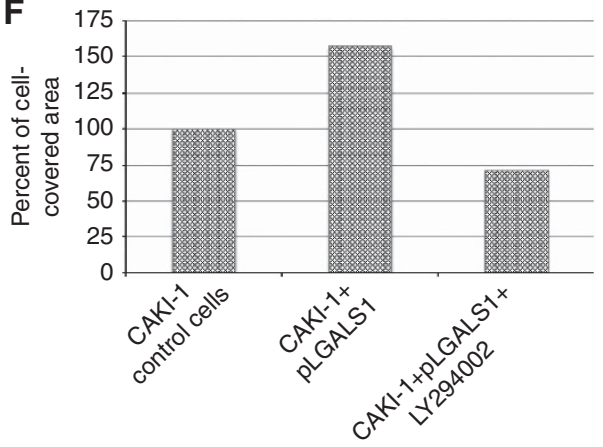

G 7000

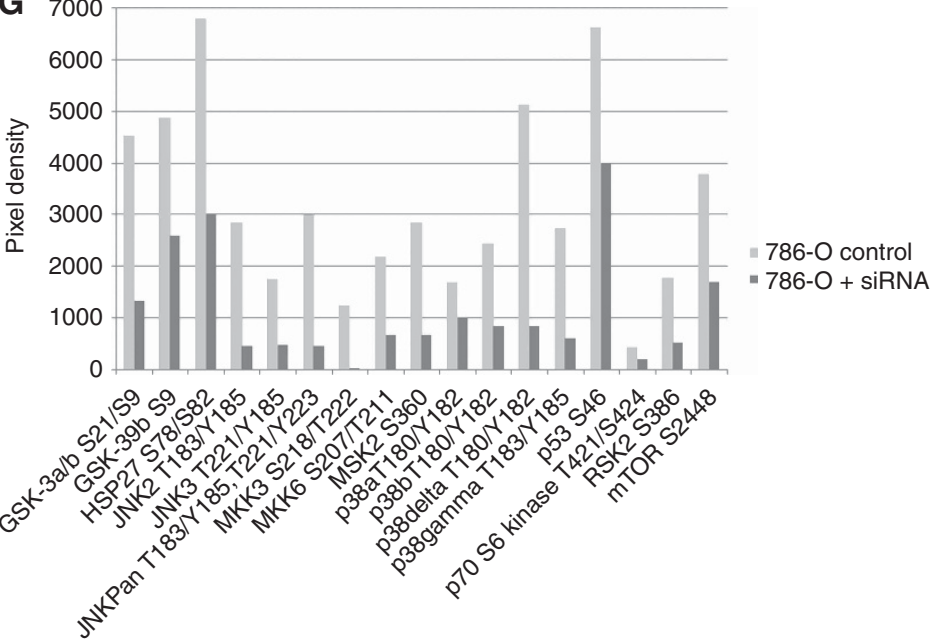

Figure 3. (A) The addition of $\mathrm{CoCl}_{2}$ to CAK-1 cells resulted in increased HIF-1 $\alpha$ protein in a dose-dependent manner of 100, 200, 300 and $400 \mu \mathrm{m}$ $\mathrm{CoCl}_{2}$. Gal-1 expression showed a parallel dose-dependent increase in expression. $\alpha$-Tubulin was used as a loading control. (B) Densitometry analysis showed HIF-1 $\alpha$ expression was increased by $4.45,7.65,8.68,14.85$ times, after the addition of 100, 200, 300 and $400 \mu \mathrm{M}$

$\mathrm{CoCl}_{2}$, respectively, whereas Gal-1 protein expression showed an increase of $6.05,11.33,11.06$ and 13.98 times, respectively. ${ }^{\star} P<0.001$.

(C) Photomicrograph of the CAKI-1 MAPK array scans. We compared the phosphorylation of MAPK proteins in CAKI-1 cells transfected with pLGALS to CAKI-1 cells transfected with the empty vector. (D) A bar graph showing pixel density of phosphorylated proteins in CAKI-1 cells before and after transfection with pLGALS1 and densitometry analysis. Protein expression was assessed using a MAPK array. A number of phosphorylated proteins were upregulated as a result of Gal-1 overexpression. Only phosphorylated proteins that had relative expression values $>1.5$ are shown in the bar graph. (E) WB showing expression of phospho-Akt (p-Akt, S473), total Akt, phospho-mTOR (p-mTOR, S2448), before and after transfection with pLGALS1. Both p-Akt and p-mTOR showed increased expression after transfection of pLGALS1. These effects were abolished with the addition of LGALS1 siRNA.,$+ 5 \mu \mathrm{m}$ siRNA;,$++ 10 \mu \mathrm{m}$ siRNA. (F) The addition of LY294002 to CAKI-1 cells transfected with pLGALS1 resulted in decreased cellular migration when compared with cells transfected with pLGALS1 alone or the control cells. (G) A bar graph showing pixel density of phosphorylated proteins before and after 786-O transfection with siRNA directed against LGALS1 on a MAPK array. Only phospho-proteins that had relative expression values $>1.5$ or $<0.67$ when compared with control cells are shown.

pLGALS1 where levels of phosphorylated Akt and mTOR returned to endogenous levels.

In order to further validate our results, we examined the effect of Akt inhibition on cellular migration in the presence and absence of Gal-1. Although CAKI-1 cells transfected with pLGALS1 showed increased cellular migration when compared with control cells, this effect was diminished after the addition of the Akt inhibitor
LY294002 (Figure 3F). These data suggest that Gal-1 mediates its biological effects through the Akt/mTOR signaling pathway.

We also assayed for the relative levels of phosphorylation of MAPKs before and after siRNA knockdown of Gal-1 using the MAPK array. Knockdown of Gal-1 resulted in decreased expression of phosphorylated proteins shown to be involved in cellular migration (Figure $3 \mathrm{G}$ and Supplementary Figure 1). These include 

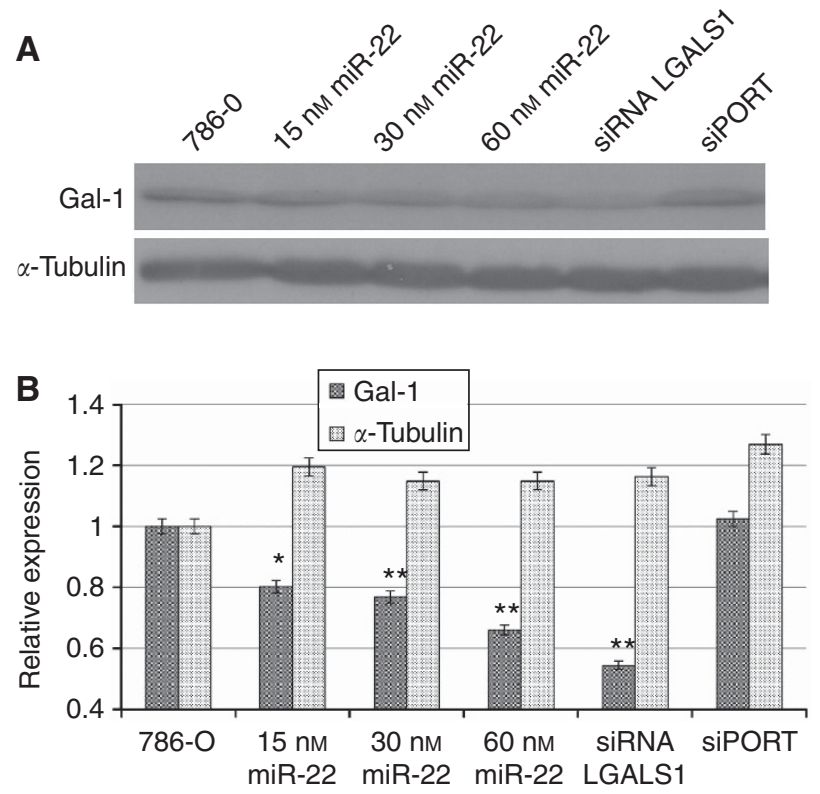

C

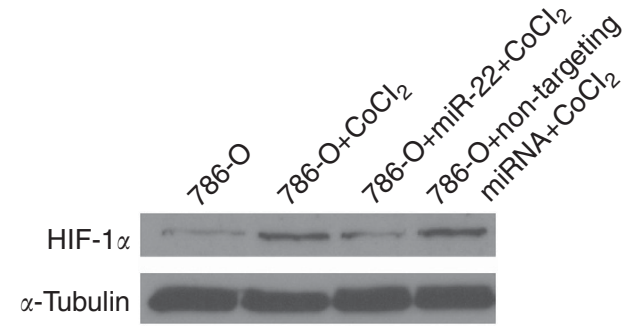

Figure 4. (A) 786-O cells transfected with increasing concentrations of miR-22 showed decreased protein expression of Gal-1.

(B) Densitometry analysis shows expression levels of Gal-1 decreased to $0.80,0.77$ and 0.66 after transfection with 15,30 and $60 \mathrm{~nm}$ of miR-22, respectively. Gal-1 expression was lowest after transfection with the LGALS1 siRNA. There was no significant difference in Gal-1 expression when cells were transfected with siPORT only when compared with control cells. ${ }^{\star} P<0.05,{ }^{*} P<0.01$. (C) The addition of $\mathrm{CoCl}_{2}$ to $786-\mathrm{O}$ cells resulted in increased HIF-1 $\alpha$ expression. Cells treated with $\mathrm{CoCl}_{2}$ and transfected with miR-22 were resistant to increases in HIF- $1 \alpha$, whereas cells co-transfected with a non-targeting miRNA displayed HIF- $1 \alpha$ expression levels that were comparable to cells treated with $\mathrm{CoCl}_{2}$ only.

HSP27, JNK, JNK2, RSK1, RSK2, TOR, p38 delta and p38 gamma. To ensure these were not off target effects, we did a MTT assay to measure cell viability and found that decreased phosphorylation was not due to decreased cell viability (data not shown).

miR-22 can target both Gal-1 and HIF-1 $\alpha$. We recently identified miRNAs dysregulated in metastatic compared with primary RCC (White et al, 2011; Khella et al, 2012). Among these, miR-22 was shown to be downregulated in RCC metastasis. Target prediction analysis identified Gal-1 as a predicted target of miR-22. We hypothesised that decreased expression of miR-22 in metastatic RCC releases its inhibitory effect and results in increased expression of Gal-1. Interestingly, miR-22 is also predicted to target HIF- $1 \alpha$. In order to validate the effect of miR-22 on Gal-1, we compared the expression level of Gal-1 before and after transfection with miR-22. Our results showed there was a dosedependent decrease in Gal-1 protein concentration with increasing concentrations of miR-22 (15, 30 and 60 nm; Figure 4A). There was no change in Gal-1 expression when cells were transfected with transfection reagent only. The maximum decrease in Gal-1

\begin{tabular}{|c|c|c|c|}
\hline & $\mathrm{HR}^{\mathrm{a}}$ & $95 \% \mathrm{Cl}^{\mathrm{b}}$ & $P$-value \\
\hline \multicolumn{4}{|l|}{ Univariate analysis } \\
\hline \multicolumn{4}{|l|}{ Galectin-1 status $^{c}$} \\
\hline Low expression & 1.00 & & \\
\hline High expression & 2.03 & $1.038-3.99$ & 0.039 \\
\hline Tumor size (ordinal) & 1.18 & $1.064-1.31$ & 0.002 \\
\hline Sex & 2.74 & $0.78-9.56$ & 0.11 \\
\hline Stage (ordinal) & 2.57 & $1.47-4.49$ & 0.001 \\
\hline \multicolumn{4}{|c|}{ Multivariate analysis $^{d}$} \\
\hline \multicolumn{4}{|l|}{ Galectin-1 status $^{c}$} \\
\hline Low expression & 1.00 & & \\
\hline High expression & 2.08 & $1.049-4.11$ & 0.036 \\
\hline Tumor Size (ordinal) & 1.13 & $0.99-1.28$ & 0.055 \\
\hline Sex & 1.65 & $0.44-6.17$ & 0.45 \\
\hline Stage (ordinal) & 1.86 & $1.00-3.45$ & 0.048 \\
\hline \multicolumn{4}{|c|}{ 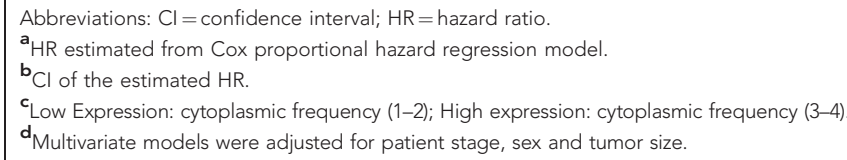 } \\
\hline
\end{tabular}

expression was seen when cells were transfected with the LGALS1 siRNA, as expected. This effect was more clear after densitometry analysis that showed Gal-1 protein expression was decreased to 0.8 $(P<0.05), 0.8(P<0.01)$ and $0.7(P<0.01)$ times after transfection with 15, 30 and $60 \mathrm{~nm}$ miR-22, respectively (Figure 4B).

We also examined the effect of miR-22 on HIF- $1 \alpha$. As expected, cells showed increased HIF- $1 \alpha$ after the addition of $\mathrm{CoCl}_{2}$ (Figure 4C). Cells that were co-transfected with $\mathrm{CoCl}_{2}$ and miR-22 were resistant to increases in HIF- $1 \alpha$, whereas cells co-transfected with a non-targeting miRNA had no effect on HIF- $1 \alpha$ expression and showed levels that were comparable to control cells where HIF- $1 \alpha$ was increased after exposure to $\mathrm{CoCl}_{2}$. Taken together, these results suggest that miR-22 can target both HIF- $1 \alpha$ and Gal-1 and may have some protective effect against hypoxia in RCC cells.

Prognostic significance of Gal-1 in RCC patients. We examined the clinical utility of Gal-1 as a prognostic marker for ccRCC patients using immunohistochemistry in 92 patients. Higher Gal-1 expression was significantly associated with tumors $\geqslant 4 \mathrm{~cm}$ when compared with tumors $\leqslant 4 \mathrm{~cm}(P=0.034$; Supplementary Table 1). Also, high levels of Gal-1 were associated with high-grade tumors (grades III/IV) when compared with low-grade (grade I or grade II) tumors $(P<0.001)$. We also assessed the clinical significance of Gal-1 protein expression for cancer recurrence. Cox hazard regression analysis showed that patients who had high Gal-1 expression had a shorter disease-free survival $(P=0.039$, $\mathrm{HR}=2.03, \mathrm{CI}=1.04-3.99$; Table 1$)$ than patients with low Gal-1 expression. In addition, multivariate analysis showed that Gal-1 expression retained its prognostic significance when controlling for other variables $(\mathrm{HR}=2.08, \mathrm{CI}=1.05-4.11)$ and was a stronger poor prognostic predictor than tumor stage $(P=0.036$ vs $P=0.048$, respectively). Furthermore, these data were verified through Kaplan-Meier analysis that showed patients with high Gal-1 expression had significantly shorter disease-free survival than patients with low Gal-1 expression ( $P=0.013$; Figure $5 \mathrm{~A})$.

In addition, we examined LGALS1 mRNA expression in an independent set of 404 patients from TCGA databases as described 

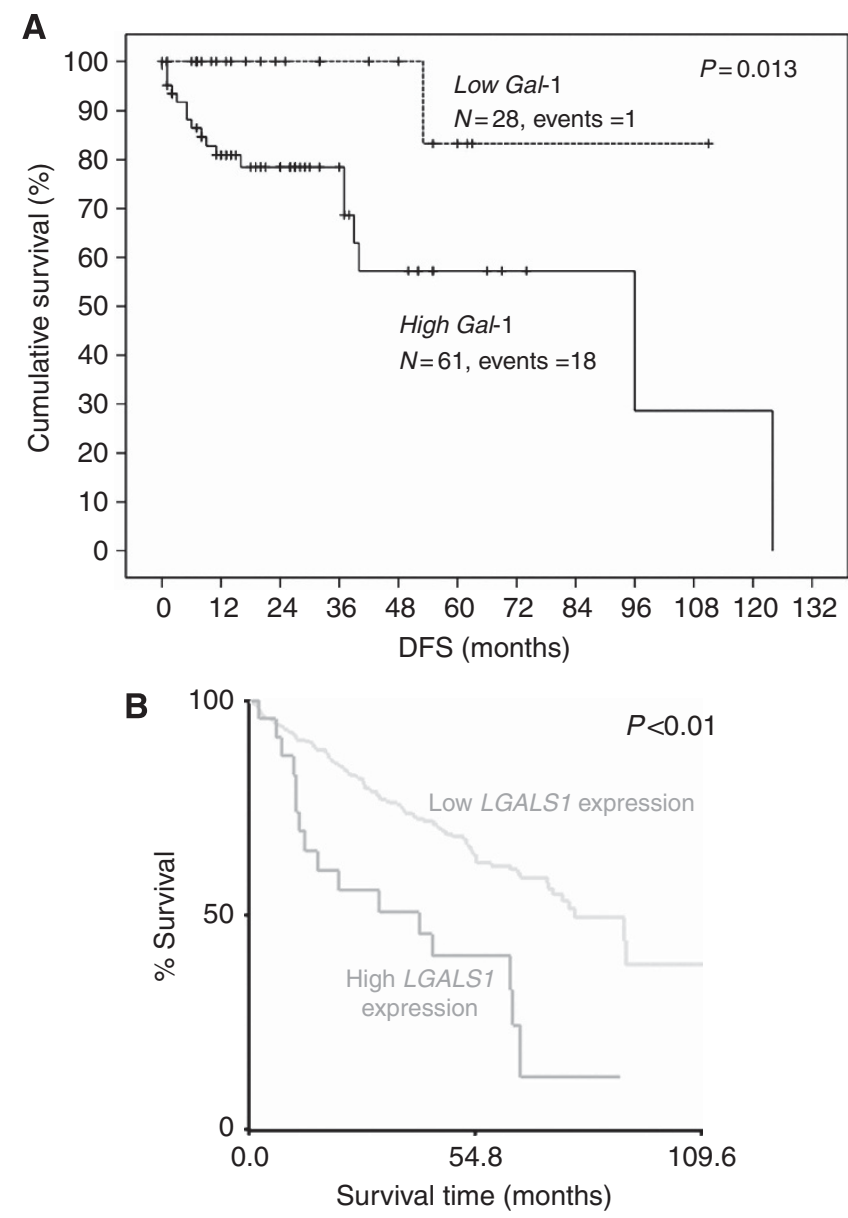

Figure 5. (A) Kaplan-Meier analysis of Gal-1 protein expression in 92 cCRCC patients showed that high Gal-1 expression is associated with a lower disease-free survival than patients with low Gal-1 expression $(P=0.013)$. (B) The same results were obtained at the mRNA level in the independent TCGA database. Using a relative expression value of 708.6 to separate patients with low and high Gal-1 expression, patients with high LGALS1 expression had significantly lower overall survival compared with those who had low LGALS1 expression (41 vs 78 months; $P<0.01$ ).

in the Materials and Methods section. LGALS1 mRNA expression was statistically significantly higher in primary ccRCC compared with normal kidney tissue from the same patient $(P<0.01)$. A cutoff value was calculated to separate patients into two groups; 'low' or 'high' Gal-1 expression. We found that patients with high LGALS1 expression in primary tumors had a significant shorter overall survival compared with patients who had low LGALS1 expression (41 vs 78 months; $P<0.01$; Figure 5B).

\section{DISCUSSION}

Our findings suggest Gal-1 is a downstream effector molecule of miR-22 and is involved in the HIF/mTOR signaling axis in RCC. Increased HIF- $1 \alpha$, because of the hypoxic tumor microenvironment, translocates to the nucleus and transcriptionally activates the LGALS1 gene (Figure 6). Gal-1, by a mechanism that has yet to be elucidated, then activates Akt by phosphorylating S473, which in turn phosphorylates mTOR (S2448) and p70S6 kinase to result in increased protein synthesis and cell migration. This pathway can be blocked by the addition of siRNA that is

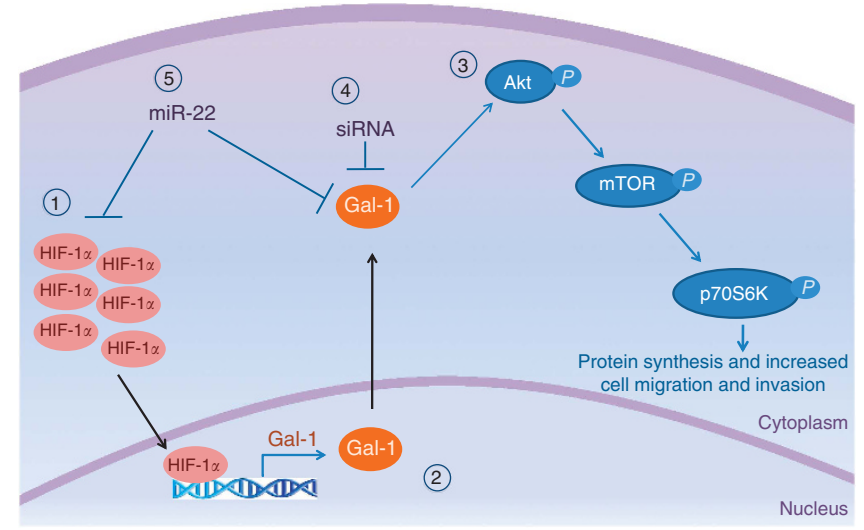

Figure 6. A schematic showing a proposed mechanism of Gal-1 involvement in the HIF/mTOR signaling axis in renal cell carcinoma. (1) Increased HIF-1 $\alpha$ because of the hypoxic tumor microenvironment (2) translocates to the nucleus and transcriptionally activates the LGALS1 gene. Gal-1 protein then, by a mechanism that has yet to be elucidated, (3) activates Akt by phosphorylating S473, which in turn phosphorylates mTOR at S2448 and p70S6 kinase to result in increased protein synthesis. This pathway can be blocked by the addition of (4) siRNA that is directed towards Gal-1. We also showed that the pathway can be affected by miRNA as (5) miR-22 can target both Gal-1 and HIF-1 $\alpha$.

directed towards Gal-1. In addition, we hypothesise that miR-22 overexpression may negatively regulate multiple targets (Gal-1 and HIF- $1 \alpha$ ) along this pathway.

Our previous work identified Gal-1 as a potential marker that is upregulated in metastatic compared with primary RCC (Masui et al, 2013). In our current work, we further explored the role of Gal-1 in tumor progression and examined its potential prognostic significance. Our results are in agreement with recent literature showing the effect of Gal-1 expression on cellular migration in glioblastoma, where Gal-1 expression directly correlated to cell migratory and invasive abilities (Jung et al, 2008). In addition, Gal-1 overexpression resulted in a significant increase in the invasive ability of cancer cells (Wu et al, 2009). Mechanistic studies showed that Gal-1 increased expression of matrix-metalloproteinases and reorganised the actin cytoskeleton.

Gal-1 was shown to be regulated by HIF-1 $\alpha$. Zhao et al (2010) showed Gal-1 can mediate HIF-1-induced migration and invasion of colon cancer cells. They identified two hypoxia-responsive elements upstream of the transcriptional start site of Gal-1 suggesting HIF-1 directly targets Gal-1. Furthermore, ectopic expression of Gal-1 was able to restore invasion and migration ability in HIF- $1 \alpha$-knocked-down colon cancer cells suggesting Gal-1 is a factor in the HIF-1-induced biological pathways. The relation of Gal-1 to HIF is interesting in kidney cancer as HIF- $1 \alpha$ has a central role in the pathogenesis of ccRCC. Approximately $70 \%$ RCC tumors have inactivated von Hippel-Lindau, which results in the accumulation of HIF- $1 \alpha$ with subsequent activation of its downstream targets and biological effects. Our results show that in RCC cells increased HIF- $1 \alpha$ expression correlates with increased Gal-1 expression and subsequently increased cellular migration and invasion (Figure 1). Also, it has been suggested that mTOR can contribute to HIF regulation through a feedback mechanism. In irradiated tumors, it was shown that the Akt/ mTOR pathway contributes to the stabilisation and accumulation of HIF-1 $\alpha$ (Harada et al, 2009).

The effect of Gal-1 on cellular migration can be mediated through the activation and phosphorylation of Akt, and further downstream mTOR. Phosphorylation of mTOR increases binding 
to raptor and is a part of the mTOR complex-1 (mTORC1) (Rosner et al, 2010), which regulates translation by activation of p70S6K and eIF-4E binding protein 1 (4E-BP1). This finding is supported by others who found Gal-1 induces mTOR phosphorylation in mouse embryonic stem cells (Lee and Han, 2008; Lee et al, 2009). Furthermore, Gal-1 was found to have an effect on Akt in trophoblast tumor cells (Fischer et al, 2011). Also, knocking down of the activity of Gal-1 resulted in decreased cellular migration and invasion (Figure 2) suggesting the potential use of Gal-1 as a therapeutic target. Similar results have been reported in prostate cancer. Laderach et al (2012) showed that silencing Gal-1 reduced tumor vascularisation without altering expression of other angiogenesis-related genes and suggested a role for Gal-1 as an attractive target for anti-angiogenic therapy in advanced stages of prostate cancer.

In the era of targeted therapies for metastatic RCC, drugs that inhibit the mTOR signaling pathway have had an important role. mTOR inhibitors, like Temsirolimus and Everolimus, are now used as targeted therapy for metastatic RCC. They bind to FKBP12 and inhibit the mTORC1, suppressing tumor growth and angiogenesis. Temsirolimus improved survival first line in poor prognostic patients (Hudes et al, 2007) and everolimus improved progressionfree survival second line (Motzer et al, 2008). The fact that the majority of tumors develop resistance to a single targeted therapy after an initial response or stability has led to combination therapy trials. With the exception of bevacizumab given with interferon (Escudier et al, 2007) targeted therapy combinations have proven either too toxic or have no added value to the patient. Our results show that Gal-1 is involved in the mTOR signaling pathway and can contribute to its activation. The effective blockage of Gal-1 in combination with an mTOR drug or a TKI antiangiogenic therapy may represent a novel approach in the treatment of kidney cancer.

Our data showed that Gal-1 gene expression was a significant predictor of poor prognosis in RCC. Our immunohistochemical analysis showed that patients with high Gal-1 expression had significantly lower disease-free survival $(P=0.0013)$. In the independent TCGA patient cohort, high LGALS1 was associated with shorter overall survival (41 vs 78 months; $P<0.01$; Figure 5) compared with those with low LGALS1 expression. Gal-1 has also been shown to be a prognostic marker in other cancers. For example, Kim et al (2012b) found that ovarian cancer patients with high Gal-1 expression had shorter progression-free survival than patients with low expression. Also, in hepatocellular carcinoma, patients with high Gal-1 expression had a significantly higher rate of tumor recurrence and shorter overall survival than those with lower Gal-1 expression (Wu et al, 2012). High level of Gal-1 expression was also shown to be a negative prognostic predictor in laryngeal squamous carcinoma (Saussez et al, 2007).

To conclude, we showed that Gal-1 is increased in metastatic RCC and can contribute to cancer-promoting biological pathways including cellular migration and invasion. We also showed the potential use of Gal-1 as a prognostic marker and therapeutic target. These need to be validated on a larger scale.

\section{ACKNOWLEDGEMENTS}

This work was supported by grants from the Canadian Cancer Society (grant \# 20185), Prostate Cancer Canada (grant \# 2010555), the Ministry of Research and Innovation of the Government of Ontario and the Kidney Foundation of Canada. NMAW is the recipient of post-doctoral fellowships from the Canadian Institutes of Health Research and Cancer Research Society.

\section{REFERENCES}

Arsanious A, Bjarnason GA, Yousef GM (2009) From bench to bedside: current and future applications of molecular profiling in renal cell carcinoma. Mol Cancer 8: 20.

Barondes SH, Castronovo V, Cooper DN, Cummings RD, Drickamer K, Feizi T, Gitt MA, Hirabayashi J, Hughes C, Kasai K (1994) Galectins: a family of animal beta-galactoside-binding lectins. Cell 76: 597-598.

Brandt B, Abou-Eladab EF, Tiedge M, Walzel H (2010) Role of the JNK/c-Jun/ AP-1 signaling pathway in galectin-1-induced T-cell death. Cell Death Dis 1: e23.

Chiang WF, Liu SY, Fang LY, Lin CN, Wu MH, Chen YC, Chen YL, Jin YT (2008) Overexpression of galectin-1 at the tumor invasion front is associated with poor prognosis in early-stage oral squamous cell carcinoma. Oral Oncol 44: 325-334.

Croci DO, Salatino M, Rubinstein N, Cerliani JP, Cavallin LE, Leung HJ, Ouyang J, Ilarregui JM, Toscano MA, Domaica CI, Croci MC, Shipp MA, Mesri EA, Albini A, Rabinovich GA (2012) Disrupting galectin-1 interactions with $\mathrm{N}$-glycans suppresses hypoxia-driven angiogenesis and tumorigenesis in Kaposi's sarcoma. J Exp Med 209: 1985-2000.

Diamandis M, White NM, Yousef GM (2010) Personalized medicine: marking a new epoch in cancer patient management. Mol Cancer Res 8: $1175-1187$.

Dihazi H, Muller C, Asif AR, Flad T, Elmaouhoub A, Muller GA (2007) Whole cell profiling and identification of galectin-1 as a potential marker of renal cell carcinoma. Proteomics Clin Appl 1: 200-214.

Escudier B, Pluzanska A, Koralewski P, Ravaud A, Bracarda S, Szczylik C, Chevreau C, Filipek M, Melichar B, Bajetta E, Gorbunova V, Bay JO, Bodrogi I, Jagiello-Gruszfeld A, Moore N (2007) Bevacizumab plus interferon alfa-2a for treatment of metastatic renal cell carcinoma: a randomised, double-blind phase III trial. Lancet 370: 2103-2111.

Fischer I, Weber M, Kuhn C, Fitzgerald JS, Schulze S, Friese K, Walzel H, Markert UR, Jeschke U (2011) Is galectin-1 a trigger for trophoblast cell fusion?: the MAP-kinase pathway and syncytium formation in trophoblast tumour cells BeWo. Mol Hum Reprod 17: 747-757.

Harada H, Itasaka S, Kizaka-Kondoh S, Shibuya K, Morinibu A, Shinomiya K, Hiraoka M (2009) The Akt/mTOR pathway assures the synthesis of HIF-1alpha protein in a glucose- and reoxygenation-dependent manner in irradiated tumors. J Biol Chem 284: 5332-5342.

Ho VT, Bunn HF (1996) Effects of transition metals on the expression of the erythropoietin gene: further evidence that the oxygen sensor is a heme protein. Biochem Biophys Res Commun 223: 175-180.

Hudes G, Carducci M, Tomczak P, Dutcher J, Figlin R, Kapoor A, Staroslawska E, Sosman J, McDermott D, Bodrogi I, Kovacevic Z, Lesovoy V, Schmidt-Wolf IG, Barbarash O, Gokmen E, O’Toole T, Lustgarten S, Moore L, Motzer RJ (2007) Temsirolimus, interferon alfa, or both for advanced renal-cell carcinoma. $N$ Engl J Med 356: 2271-2281.

Jung EJ, Moon HG, Cho BI, Jeong CY, Joo YT, Lee YJ, Hong SC, Choi SK, Ha WS, Kim JW, Lee CW, Lee JS, Park ST (2007) Galectin-1 expression in cancer-associated stromal cells correlates tumor invasiveness and tumor progression in breast cancer. Int J Cancer 120: 2331-2338.

Jung TY, Jung S, Ryu HH, Jeong YI, Jin YH, Jin SG, Kim IY, Kang SS, Kim HS (2008) Role of galectin-1 in migration and invasion of human glioblastoma multiforme cell lines. J Neurosurg 109: 273-284.

Khella HW, White NM, Faragalla H, Gabril M, Boazak M, Dorian D, Khalil B, Antonios H, Bao TT, Pasic MD, Honey RJ, Stewart R, Pace KT, Bjarnason GA, Jewett MA, Yousef GM (2012) Exploring the role of miRNAs in renal cell carcinoma progression and metastasis through bioinformatic and experimental analyses. Tumour Biol 33: 131-140.

Kim HJ, Do IG, Jeon HK, Cho YJ, Park YA, Choi JJ, Sung CO, Lee YY, Choi CH, Kim TJ, Kim BG, Lee JW, Bae DS (2012a) Galectin 1 expression is associated with tumor invasion and metastasis in stage IB to IIA cervical cancer. Hum Pathol 44(1): 62-68.

Kim HJ, Jeon HK, Cho YJ, Park YA, Choi JJ, Do IG, Song SY, Lee YY, Choi CH, Kim TJ, Bae DS, Lee JW, Kim BG (2012b) High galectin-1 expression correlates with poor prognosis and is involved in epithelial ovarian cancer proliferation and invasion. Eur J Cancer 48: 1914-1921.

Laderach DJ, Gentilini L, Giribaldi L, Delgado VC, Nugnes L, Croci DO, Al NN, Sacca P, Casas G, Mazza O, Shipp MA, Vazquez ES, Chauchereau A, Kutok JL, Rodig SJ, Elola MT, Compagno D, Rabinovich GA (2012) A unique galectin signature in human prostate cancer progression suggests 
galectin-1 as a key target for treatment of advanced disease. Cancer Res 73(1): 86-96.

Lee MY, Han HJ (2008) Galectin-1 upregulates glucose transporter-1 expression level via protein kinase C, phosphoinositol-3 kinase, and mammalian target of rapamycin pathways in mouse embryonic stem cells. Int J Biochem Cell Biol 40: 2421-2430.

Lee MY, Lee SH, Park JH, Han HJ (2009) Interaction of galectin-1 with caveolae induces mouse embryonic stem cell proliferation through the Src, ERas, Akt and mTOR signaling pathways. Cell Mol Life Sci 66: 1467-1478.

Masui O, White NMA, DeSouza LV, Krakovska O, Matta A, Metias S, Khalil B, Romaschin AD, Honey RJ, Stewart R, Pace K, Bjarnason GA, Siu KW, Yousef GM (2013) Quantitative proteomic analysis in metastatic renal cell carcinoma reveals a unique set of proteins with potential prognostic significance. Mol Cell Proteomics 12: 132-144.

Motzer RJ, Escudier B, Oudard S, Hutson TE, Porta C, Bracarda S, Grunwald V, Thompson JA, Figlin RA, Hollaender N, Urbanowitz G, Berg WJ, Kay A, Lebwohl D, Ravaud A (2008) Efficacy of everolimus in advanced renal cell carcinoma: a double-blind, randomised, placebo-controlled phase III trial. Lancet 372: 449-456.

Ralhan R, DeSouza LV, Matta A, Chandra TS, Ghanny S, Datta GS, Bahadur S, Siu KW (2008) Discovery and verification of head-and-neck cancer biomarkers by differential protein expression analysis using iTRAQ labeling, multidimensional liquid chromatography, and tandem mass spectrometry. Mol Cell Proteomics 7: 1162-1173.

Romaniuk MA, Croci DO, Lapponi MJ, Tribulatti MV, Negrotto S, Poirier F, Campetella O, Rabinovich GA, Schattner M (2012) Binding of galectin-1 to alphaIIbbeta(3) integrin triggers 'outside-in' signals, stimulates platelet activation, and controls primary hemostasis. FASEB J 26: 2788-2798.

Rosner M, Siegel N, Valli A, Fuchs C, Hengstschlager M (2010) mTOR phosphorylated at S2448 binds to raptor and rictor. Amino Acids 38: 223-228.

Saussez S, Decaestecker C, Lorfevre F, Cucu DR, Mortuaire G, Chevalier D, Wacreniez A, Kaltner H, Andre S, Toubeau G, Camby I, Gabius HJ, Kiss R (2007) High level of galectin-1 expression is a negative prognostic predictor of recurrence in laryngeal squamous cell carcinomas. Int $J$ Oncol 30: 1109-1117.

van den Brule F, Califice S, Garnier F, Fernandez PL, Berchuck A, Castronovo V (2003) Galectin-1 accumulation in the ovary carcinoma peritumoral stroma is induced by ovary carcinoma cells and affects both cancer cell proliferation and adhesion to laminin-1 and fibronectin. Lab Invest 83: $377-386$.

White NM, Khella HW, Grigull J, Adzovic S, Youssef YM, Honey RJ, Stewart R, Pace KT, Bjarnason GA, Jewett MA, Evans AJ, Gabril M, Yousef GM (2011) miRNA profiling in metastatic renal cell carcinoma reveals a tumour-suppressor effect for miR-215. Br J Cancer 105: 1741-1749.

White NM, Yousef GM (2011) Translating molecular signatures of renal cell carcinoma into clinical practice. J Urol 186: 9-11.

Wu H, Chen P, Liao R, Li YW, Yi Y, Wang JX, Sun TW, Zhou J, Shi YH, Yang XR, Jin JJ, Cheng YF, Fan J, Qiu SJ (2012) Overexpression of galectin-1 associates with poor prognosis in human hepatocellular carcinoma following resection. J Gastroenterol Hepatol 27(8): 1312-1319.

Wu MH, Hong TM, Cheng HW, Pan SH, Liang YR, Hong HC, Chiang WF, Wong TY, Shieh DB, Shiau AL, Jin YT, Chen YL (2009) Galectin-1mediated tumor invasion and metastasis, up-regulated matrix metalloproteinase expression, and reorganized actin cytoskeletons. Mol Cancer Res 7: 311-318.

Zhao XY, Chen TT, Xia L, Guo M, Xu Y, Yue F, Jiang Y, Chen GQ, Zhao KW (2010) Hypoxia inducible factor-1 mediates expression of galectin-1: the potential role in migration/invasion of colorectal cancer cells. Carcinogenesis 31: 1367-1375.

This work is published under the standard license to publish agreement. After 12 months the work will become freely available and the license terms will switch to a Creative Commons AttributionNonCommercial-Share Alike 3.0 Unported License.

Supplementary Information accompanies this paper on British Journal of Cancer website (http://www.nature.com/bjc) 